\title{
Extracellular NAD and ATP: Partners in immune cell modulation
}

\author{
Friedrich Haag • Sahil Adriouch • Anette Braß • \\ Caroline Jung • Sina Möller • Felix Scheuplein • \\ Peter Bannas • Michel Seman • Friedrich Koch-Nolte
}

Received: 8 February 2006 / Accepted: 22 October 2006 / Published online: 9 January 2007

(C) Springer Science + Business Media B.V. 2007

\begin{abstract}
Extracellular NAD and ATP exert multiple, partially overlapping effects on immune cells. Catabolism of both nucleotides by extracellular enzymes keeps extracellular concentrations low under steady-state conditions and generates metabolites that are themselves signal transducers. ATP and its metabolites signal through purinergic P2 and P1 receptors, whereas extracellular NAD exerts its effects by serving as a substrate for ADP-ribosyltransferases (ARTs) and NAD glycohydrolases/ADPR cyclases like CD38 and CD157. Both nucleotides activate the P2X7 purinoceptor, although by different mechanisms and with different characteristics. While ATP activates P2X7 directly as a soluble ligand, activation via NAD occurs by ART-dependent ADPribosylation of cell surface proteins, providing an immobilised ligand. P2X7 activation by either route leads to phosphatidylserine exposure, shedding of CD62L, and ultimately to cell death. Activation by ATP requires high micromolar concentrations of nucleotide and is readily reversible, whereas NAD-dependent stimulation begins at low micromolar concentrations and is more stable. Under conditions of cell stress or inflammation, ATP and NAD are released into the extracellular space from intracellular stores by lytic and non-lytic mechanisms, and may serve as "danger signals" to alert the immune response to tissue damage. Since ART expression is limited to naïve/resting T cells, P2X7-mediated NAD-induced cell death (NICD) specifically targets this cell population. In
\end{abstract}

F. Haag $(\bowtie) \cdot S$. Adriouch $\cdot$ A. Braß $\cdot$ C. Jung $\cdot$ S. Möller $\cdot$

F. Scheuplein $\cdot$ P. Bannas $\cdot$ F. Koch-Nolte

Institute of Immunology, University Hospital,

Martinistr. 52, 20246 Hamburg, Germany

e-mail: haag@uke.uni-hamburg.de

F. Haag $\cdot$ S. Adriouch $\cdot$ M. Seman

INSERM U519- EA1556, Faculté de Médecine et de Pharmacie,

Université de Rouen,

F-76183 Rouen Cedex, France inflamed tissue, NICD may inhibit bystander activation of unprimed $\mathrm{T}$ cells, reducing the risk of autoimmunity. In draining lymph nodes, NICD may eliminate regulatory T cells or provide space for the preferential expansion of primed cells, and thus help to augment an immune response.

Key words ADP-ribosylation - ADP-Ribosyltransferases . apoptosis · ATP - ectoenzymes · extracellular purines .

$\mathrm{NAD} \cdot$ posttranslational protein modification

\begin{tabular}{|c|c|}
\hline \multicolumn{2}{|c|}{ Abbreviations } \\
\hline $\mathrm{ADP}$ & adenosine diphosphate \\
\hline ADPR & Adenosine diphosphate ribose \\
\hline AMP & Adenosine monophosphate \\
\hline ART & ADP-ribosyltransferase \\
\hline ATP & Adenosine triphosphate \\
\hline E-NPP & $\begin{array}{l}\text { Ecto-nucleotide pyrophosphatase/ } \\
\text { phosphodiesterase }\end{array}$ \\
\hline E-NTPD & $\begin{array}{l}\text { Ecto-nucleoside triphosphate } \\
\text { diphosphohydrolase }\end{array}$ \\
\hline FoxP3 & Forkhead box P3 \\
\hline NAADP & Nicotinic acid adenine dinucleotide phosphate \\
\hline NAD & Nicotinamide adenine dinucleotide \\
\hline NADP & Nicotinamide adenine dinucleotide phosphate \\
\hline NICD & NAD-induced cell death \\
\hline PARP & Poly(ADP-ribose) polymerase \\
\hline PS & Phosphatidyl serine \\
\hline
\end{tabular}

ATP and NAD in the extracellular compartment: From their release to the induction of specific signalling

ATP and NAD + are classic intracellular metabolites with center-stage roles in energy metabolism and electron transfer. In recent years, it has become evident that these 
purine nucleotides play important roles also in the extracellular environment, i.e., as substrates for a flurry of nucleotide-metabolising ectoenzymes, and, in the case of ATP, also as a ligand for cell surface receptors (Figs. 1 and 2).

Biosynthesis of NAD presumably takes place in several locations in the cell [1]. Under physiological conditions most (more than $70 \%$ ) of the cellular NAD content is stored and is utilised in the mitochondria primarily for metabolic purposes. In the cytoplasm and in the nucleus NAD serves cell signalling functions, as a precursor for calcium mobilising metabolites and as a substrate for two families of nuclear enzymes, i.e., poly-ADP-ribosyl polymerases (PARPs) and the sirtuin (homologues of the yeast "silent mating type information regulation 2" ( Sir2) gene) family of NAD-dependant lysine deacetylases, both of which play important roles in coordinating DNA repair, regulating transcription levels and controlling progression towards apoptosis [2-4]. Under pathophysiological conditions, such as ischemia, oxidative stress or DNA-damaging agents, cells release their mitochondrial NAD content to the cytoplasm and the nucleus by still unknown mechanisms [4]. It is not surprising, then, that NAD plays an essential role in the cellular response to stress.

Similarly, following the induction of cellular stress part of this cellular content of NAD and ATP may be released into the extracellular space. This may occur by several mechanisms involving active exocytosis or diffusion through transmembrane transporters in living cells or passive leakage across the membrane in dying cells [5-7]. Of note, release of purines by injured or dying cells has recently been suggested to serve as a "danger signal" that may alert the immune system to tissue damage [8-10].

\section{Immune modulation by extracellular ATP}

Once released, extracellular ATP and NAD can be degraded into further metabolites such as ADP, AMP or adenosine by extracellular enzymes, i.e., ecto-nucleoside triphosphate diphosphohydrolase (E-NTPDs), ecto-nucleotide pyrophosphatase/phosphodiesterase (E-NPPs), and the ecto-5'-nucleotidase CD73 (Figs. 1, 2). ATP or its by-products activate different members of the purinoceptor family of receptors. Purinoceptors comprise adenosine-sensitive P1 receptors (A1, A2a, A2b, and A3) and $\mathrm{P} 2$ receptors, which are activated by ATP, ADP, UTP, UDP or UDP-glucose [11, 12] (and NAD, see note added in proof). $P 2$ receptors are further divided into two groups: the $G$ protein-coupled seven-transmembrane $\mathrm{P} 2 \mathrm{Y}$ receptors $(\mathrm{P} 2 \mathrm{Y} 1,-2,-4,-6,-11$, $-12,-13,-14)$, and the $\mathrm{P} 2 \mathrm{X}$ ligand-gated ion channels (P2X1-7) [13, 14]. Triggering of purinoceptors by their ligands regulates important physiological functions such as platelet aggregation, local regulation of blood pressure, modulation of cardiac functions in ischemic conditions or regulation of the development of inflammation $[11,15,16]$.

Regulation of immune functions by ATP and its metabolites has been reviewed elsewhere $[8,10]$. ATP can in principle transmit signals through several different receptors, including the complete $\mathrm{P} 2 \mathrm{X}$ family and a subgroup of P2Y receptors (P2Y1, 2, 11, 12, 13) [12]. These receptors differ greatly in their relative sensitivities to ATP, with EC50s in the nanomolar (P2Y), low micromolar
Fig. 1 Chemical structure of ATP and NAD, and sites of cleavage by different ecto-enzymes

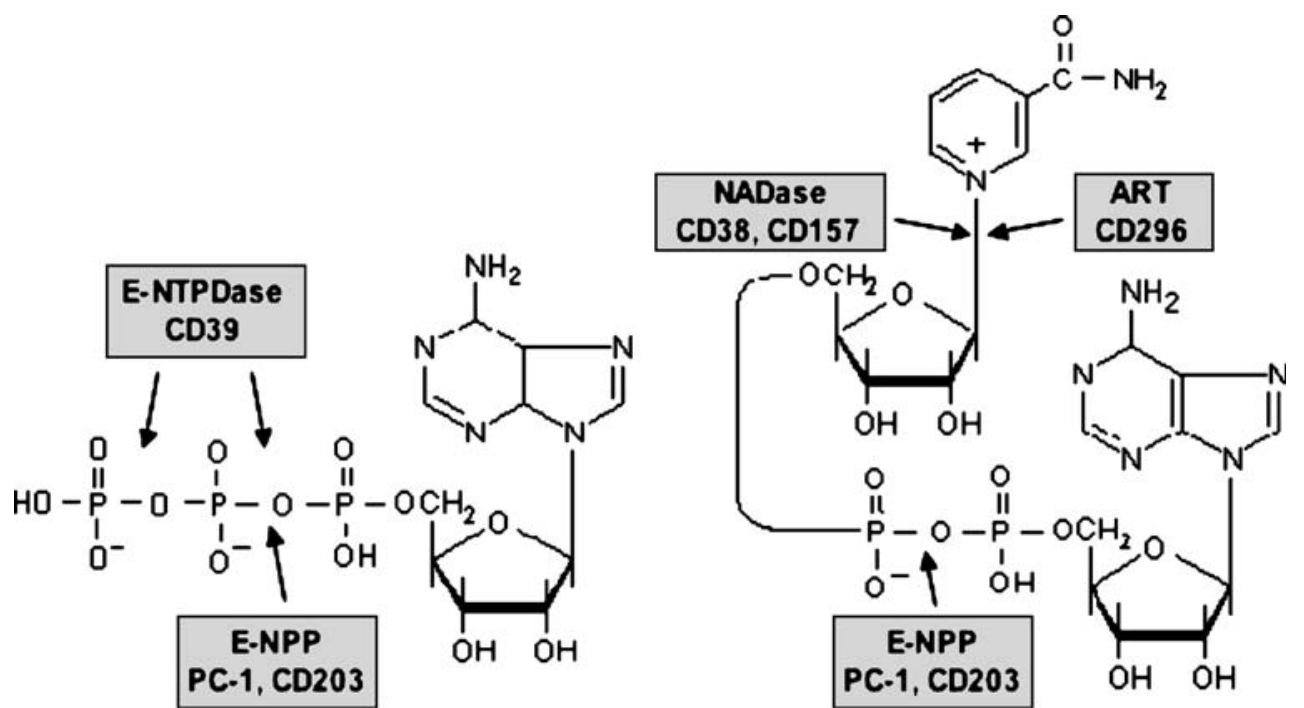

ATP 


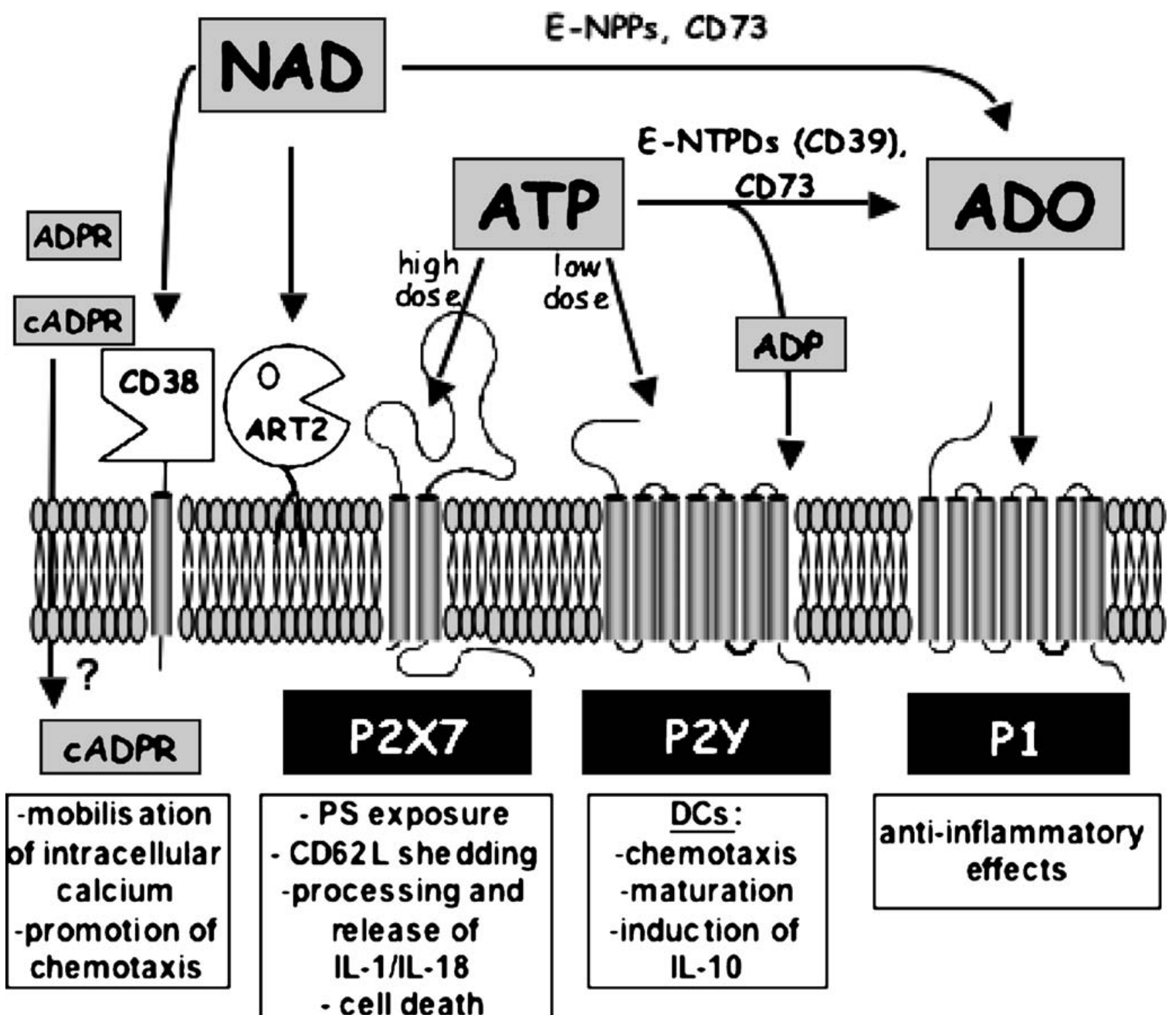

Fig. 2 Action of extracellular ATP and NAD and their metabolites on different cell surface receptors. Extracellular ATP present in high, intermediate, or low concentrations can activate $\mathrm{P} 2 \mathrm{X} 7$, other $\mathrm{P} 2 \mathrm{X}$, or $\mathrm{P} 2 \mathrm{Y}$ receptors, respectively, or is hydrolysed by the sequential action of ecto-nucleoside triphosphate diphosphohydrolases (E-NTPDs) such as CD39 and ecto-5'-nucleotidase (CD73) to ADP and adenosine (ADO). For clarity, $\mathrm{P} 2 \mathrm{X}$ receptors other than $\mathrm{P} 2 \mathrm{X} 7$ are not shown, since their presence on immune cells is not well documented. ADP can act on $\mathrm{P} 2 \mathrm{Y}$ receptors, and adenosine can activate $\mathrm{G}$ protein-coupled P1

(most P2X) or high micromolar (P2X7) ranges [8]. The situation is further complicated by the fact that extracellular ATP is rapidly metabolised, and its break-down products, notably ADP and adenosine, have signalling functions of their own through different receptors. Both pro- and antiinflammatory effects of ATP on immune cells have been reported, depending on the cell type and the available concentration of ATP. In general, P2X7, which requires high ATP concentrations acting for a short time, mediates

receptors. Extracellular NAD serves as a substrate for cell-surface ADP-ribosyltransferases (ART2), or is hydrolyzed to ADPribose by CD38. CD38 can also synthesise cyclic ADP-ribose, a known intracellular calcium mobilising agent. It is not known how cADPR gains access to the intracellular compartment. NAD (and ATP) may also be hydrolysed by ecto-nucleotide pyrophosphatase/phosphodiesterases (E-NPPs) to AMP, which in turn is hydrolysed by CD73 to adenosine. See text for details

mainly pro-inflammatory effects, such as the processing and release of interleukin- (IL-) 1 and IL-18 [17, 18], in dendritic cells and macrophages, and induces cell death in $\mathrm{T}$ cells. Activation of $\mathrm{P} 2 \mathrm{X} 7$ also stimulates the production of tumor necrosis factor alpha (TNFa) in microglial cells [19]. Low concentrations of ATP present during the maturation of DCs reduce their capacity to induce Th1typical responses in primed $\mathrm{T}$ cells $[20,21]$. These antiinflammatory effects may be due either to direct action on 


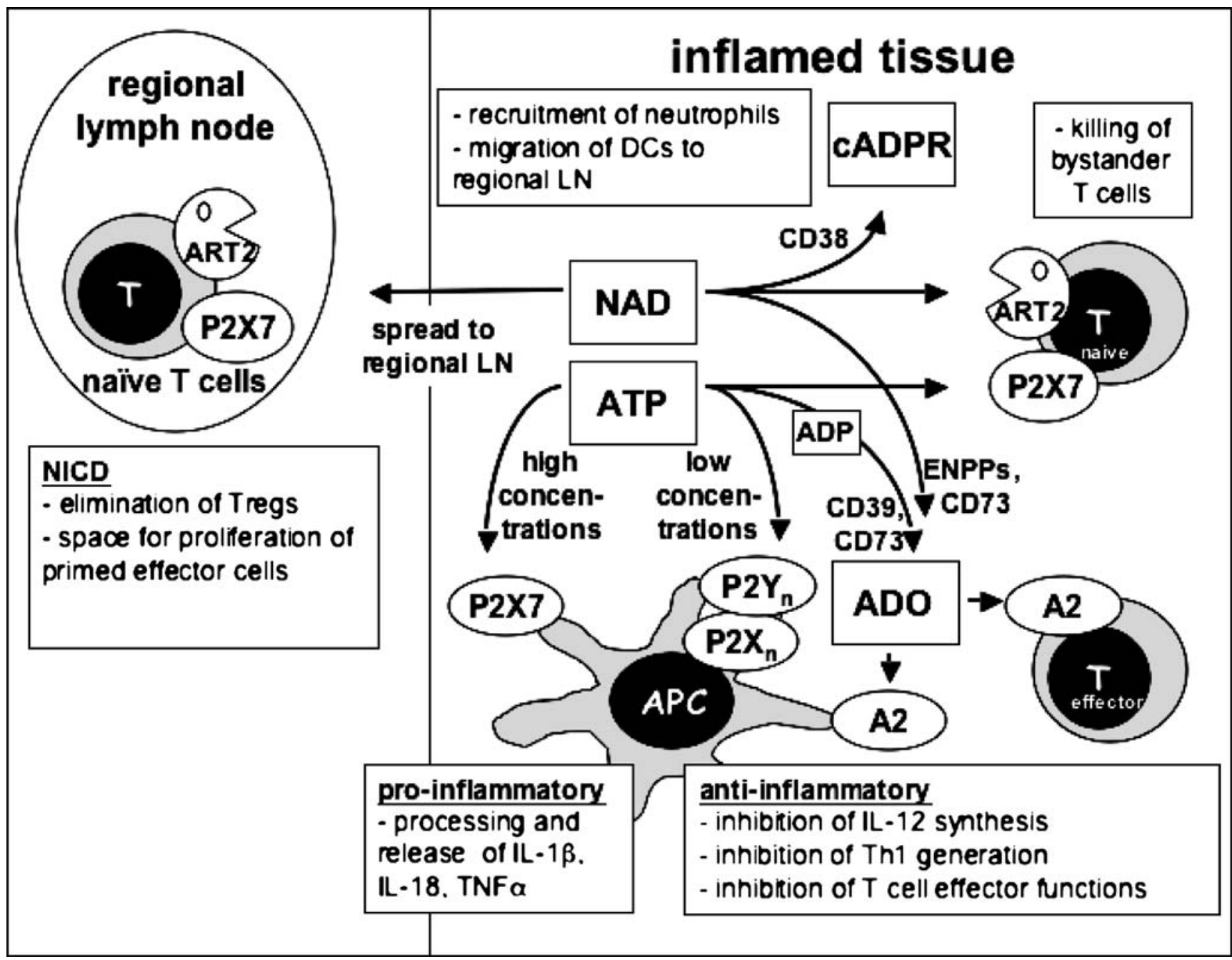

Fig. 3 Hypothetical scheme of the interplay of purine sensors during an immune response. ATP and NAD are released locally at sites of tissue injury or inflammation. At high concentrations, ATP acts on the P2X7R receptor to exert pro-inflammatory effects on antigen presenting cells or to kill $\mathrm{T}$ cells; at low concentrations it acts on other P2 receptors to downregulate the initiation of Th1 responses. NAD is used by ARTs on T cells to activate $\mathrm{P} 2 \mathrm{X} 7$, or by $\mathrm{CD} 38$ to generate cyclic

some P2Y receptors like P2Y11, or by adenosine signalling through P1 receptors (Figs. 2 and 3).

Immune modulation by extracellular NAD

Similar to ATP, NAD is also degraded in the extracellular compartment, giving rise to the generation of metabolites like cyclic ADP-ribose or adenosine that are active signal transducers (Fig. 2). In contrast to ATP, signalling through intact NAD does not involve specific membrane receptors (see note added in proof). Nevertheless, NAD may regulate cellular functions through two known enzyme families. The first family, comprising CD38 and the functionally related
ADP-ribose. It is conceivable that NAD may exert distant effects by reaching lymph nodes draining inflammatory sites in physiologically relevant concentrations. Both ATP and NAD are degraded by metabolising enyzmes to yield other signalling molecules, notably adenosine (ADO), which exerts predominantly anti-inflammatory effects through P1 receptors of the A2-subfamily. See text for details

CD157 enzyme, possesses NAD-glycohydrolase and ADPribose cyclase activities. They catalyse cleavage of NAD into ADP-ribose or cyclic ADP-ribose and nicotinamide [22], as well as the transglycosidation of NADP and nicotinic acid to yield NAADP [23]. Cyclic ADP-ribose and NAADP are newly recognised second messenger molecules, which trigger calcium release from IP3-independent intracellular stores, and which may thus play important regulatory roles $[24,25]$. However, it is controversial whether these second messengers are generated by extracellular CD38 and are then translocated to the cytosol by hitherto unknown mechanisms, or whether they are generated from intracellular NAD by an intracellular isoform of $\mathrm{CD} 38$. CD38 may also be involved in the 
regulation of immune functions by limiting the substrate availability for ADP-ribosyltransferases (see below) [26]. Mice lacking the CD38 glycohydrolase/ADP-ribosyl cyclase show an impaired antibody response to T-cell dependent antigens [27], which may be due to a defect in the migratory capacity of dendritic cells [28].

The second family of enzymes mediating signalling by NAD comprises the mono(ADP-ribosyl)transferases (ARTs), which are structurally related to ADP-ribosylating bacterial toxins. These enzymes catalyse a posttranslational modification of proteins by transferring the ADP-ribose moiety from NAD to specific amino acids, e.g., arginine residues, on target proteins [29]. This family contains five known mammalian members, ART1-ART5, which are GPIanchored membrane proteins (ART1-ART4) or secreted enzymes (ART5) [30]. Human ART1 was recently assigned the CD number CD296 [31]; it is expressed by activated granulocytes as well as by skeletal muscle, heart, and epithelial cells [32-34]. ART4 has been identified as the carrier of the Dombrock blood group alloantigens and was recently assigned the CD number CD297 [35]. ART4 is expressed prominently by erythrocytes and at lower levels also on monocytes and splenic macrophages. Only ART1, ART2 and ART5 have been shown so far to possess arginine-specific activity, while ART3 and ART4 may have acquired a new target specificity. Akin to the well-known phosphorylation reaction, posttranslational protein modification by ADP-ribosylation regulates (inhibits or activates) the functions of target proteins $[30,36]$. The ART enzyme family members thus represent new players in the epigenetic regulation of protein functions.

It has been shown that ART2, like many other GPIanchored proteins, is segregated into specialised cholesterol- and ganglioside-enriched microdomains on the cell surface termed lipid rafts [37]. Localisation into lipid rafts is important for the activity of ART2, presumably by focussing it on its target molecules. Indeed, substantial fractions of two known non-GPI-anchored target proteins of ART2, i.e., LFA-1 and P2X7, may also be recruited into lipid rafts [37].

\section{Purine sensors on cells of the immune system}

Cells of the immune system express a variety of purine sensors on their surfaces, either as purinoreceptors or as ecto-enzymes that metabolise purine nucleotides (Fig. 3). For many of the molecules, a detailed expression analysis is still hampered by a lack of suitable antibodies.

The only ATP-sensitive purinoreceptor that has been positively identified on peripheral $\mathrm{T}$ cells to date is $\mathrm{P} 2 \mathrm{X} 7$. In these cells, P2X7 mediates ATP- and NAD-dependent phosphatidyl serine (PS) exposure, CD62L shedding, and ultimately cell death $[38,39]$. Of the $\mathrm{P} 2 \mathrm{Y}$ receptors, $\mathrm{P} 2 \mathrm{Y} 6$ and P2Y14 have been described on T cells [40, 41], but these receptors are sensitive to UDP and UDP-glucose, respectively.

P2X7 is also expressed on antigen-presenting cells, including dendritic cells and macrophages, where it mediates release of the non-classically secreted cytokines IL-1 $\beta$ and IL-18 $[18,42]$, and promotes phagosome/ lysosome fusion [43-45]. P2X7 is not expressed on resting $\mathrm{B}$ cells in the mouse, but in the human has been identified on a subset of chronic B-cell lymphomas (B-CLL) [46, 47]. Immature dendritic cells also express the P2Y11 receptor [48]. This receptor, which is sensitive to nanomolar concentrations of ATP (see note added in proof), has been implicated in several responses of DCs to ATP. Low doses of ATP synergise with other stimuli like TNFa or LPS to enhance DC maturation, but the net effect is to reduce the production of IL-12p70 and increase the production of IL10 [49]. As is the case for the anti-inflammatory A2 subgroup of P1 receptors (see below), stimulation of P2Y11 causes an elevation of intracellular cAMP in DCs, which mediates its effects on DC maturation [48]. Using a different biochemical pathway, P2Y11 also inhibits the migratory response of immature DCs to chemotactic gradients, causing these cells to remain longer at sites of tissue damage [50].

The $\mathrm{G}$ protein-coupled $\mathrm{P} 1$ receptors fall into two functional groups, which serve to lower (A1 and A3 receptors) or to increase (A2a and $\mathrm{A} 2 \mathrm{~b}$ receptors) intracellular levels of cyclic AMP (cAMP). A1/A3 receptors are expressed on immature DCs, where they induce calcium flux and promote chemotaxis [51, 52]. A2a/b receptors down-regulate the production of IL-12 in LPS-matured DCs and thus inhibit the differentiation of naive CD4+ T cells towards a Th1 phenotype. T cells also express A2a receptors. Stimulation of these receptors by adenosine inhibits TCR-mediated $\mathrm{T}$ cell proliferation and upregulation of the IL-2 receptor, as well as most of the effector functions of cytotoxic $\mathrm{T}$ cells $[53,54]$. The $\mathrm{A} 2 \mathrm{a} / \mathrm{b}$ receptors are the most prominent $\mathrm{P} 1$ receptors on immune cells, and are responsible for the dominant anti-inflammatory effects of adenosine on the immune system (recently reviewed in [55]). Nucleotide-metabolising enzymes are widely distributed among cells of the immune system. Ecto-ATPase and 5 '-nucleotidase activities, which are sufficient to convert ATP into adenosine, are found on many lymphocytes and antigen presenting cells. It is worth noting that ectoadenylate kinase, the enzyme catalysing the reverse pathway, i.e., the generation of ATP from extracellular adenosine, is also present on the surface of lymphocytes [56]. Adenosine can also be generated from extracellular NAD by the sequential action of E-NPPs and 5'-nucleotidase. Although the expression of E-NPPs on immune cells 
has not been studied in detail, it is of note that E-NPP1, also known as PC-1, was originally identified as a marker for plasma cells. Finally, immune cells express NAD-dependent NADase and ADP-ribosyltransferase activities. In the mouse, the CD38 NADase/ADPR-cyclase is expressed on $\mathrm{B}$ cells and other antigen-presenting cells, and on activated, but not resting T cells [26, 27]. Conversely, the ART2 ADPribosyltransferases are expressed on resting mouse $\mathrm{T}$ cells, but not on activated ones or on antigen-presenting cells.

\section{The biological functions of ARTs and their substrate, NAD, in immune regulation}

Extracellular NAD selectively induces apoptosis of mouse $\mathrm{T}$ cells (both CD4+ and CD8+), but not of B cells [39]. Interestingly, apoptosis is observed with NAD concentrations as low as 1 micromolar. Furthermore, sensitivity to NAD is dependent on the activation state of lymphocytes. Indeed, in vitro stimulation of $\mathrm{T}$ cells with mitogens prior to NAD incubation results in relative insensitivity to NADinduced apoptosis. Similarly, in vivo-activated cells, present in freshly isolated T-cell preparations, do not respond to NAD treatment, resulting in enrichment of CD44high, CD62Llow activated /memory $\mathrm{T}$ cells in the surviving fraction. Thus, NAD selectively induces apoptosis of naive mouse $\mathrm{T}$ lymphocytes [57]. It has recently been shown that sensitivity to NAD-induced cell death is especially high in CD4+/CD25+ regulatory $\mathrm{T}$ cells expressing the transcription factor forkhead box P3 (FoxP3) [58].

What is the molecular mechanism underlying NADinduced apoptosis? Apart from NAD, none of the structurally related molecules tested (i.e., nucleosides, nucleotides or products of NAD metabolism) induced apoptosis in the micromolar range. Therefore, NAD must induce apoptosis through direct interaction with membrane proteins like ARTs, which are able to use extracellular NAD. Consistent with this interpretation, ART2 knock-out mice are completely resistant to NAD-induced apoptosis [59]. However, although C57BL/6 mice express high levels of ART2, T lymphocytes from this strain are relatively resistant to the effects of NAD. Therefore, ART2.2 is required, but not sufficient to account for NAD-induced apoptosis, and another essential factor must be involved in the process [57].

This downstream effector was identified by pharmacological studies [39]. Etheno-NAD, an NAD analogue modified in the adenine moiety, can be used as a substrate by ART2 resulting in etheno-ADP-ribosylation of target proteins [60]. However, etheno-NAD, like the NAD analogues NHD or NGD, was unable to induce apoptosis. Furthermore, pre-treatment with etheno-NAD, NHD or NGD prevented subsequent NAD-induced apoptosis. These data implied that the downstream effector was sensitive to the modification of NAD in the adenine group, a property known to hold true for purinoceptors. As adenosine itself was unable to trigger apoptosis of T lymphocytes, it was poorly conceivable that the effector belonged to the $\mathrm{P} 1$ purinoceptors. Conversely, the fact that a high dose of ATP is able to induce apoptosis of $\mathrm{T}$ cells suggested that $\mathrm{P} 2$ purinoceptors could be involved. Within the P2 receptor family, P2X7 was a good candidate, because, firstly, P2X7 is expressed on $\mathrm{T}$ lymphocytes and, secondly, because $\mathrm{P} 2 \mathrm{X} 7$ triggering is known to induce apoptosis $[61,62]$. Several lines of evidence demonstrated that P2X7 does indeed mediate NAD-induced apoptosis as a consequence of ADP-ribosylation of membrane proteins by ART2. Foremost, pharmacological studies showed that the P2X7 inhibitors KN-62 and oATP block NAD-induced apoptosis. Furthermore NAD, like ATP, was able to induce other known effects characteristic of $\mathrm{P} 2 \mathrm{X} 7$ activation, such as the formation of non-selective membrane pores permeable to large molecules up to $900 \mathrm{Da}$, the exposition of PS on the outer leaflet of the cell membrane, and the shedding of CD62L. Moreover NAD, like ATP, induced calcium uptake that could be inhibited by the P2X7 inhibitor KN-62. Finally, the generation of antibodies directed against mouse P2X7 allowed us to show that P2X7 is ADP-ribosylated in the presence of NAD and is, therefore, directly targeted by ART2 [39].

Further confirmation of the involvement of P2X7 in NAD-induced apoptosis was brought by examination of the gene coding for $\mathrm{P} 2 \mathrm{X} 7$ in $\mathrm{C} 57 \mathrm{BL} / 6$ mice. Cloning and sequencing of the P2rx7 gene showed that these mice harbour an inactivating mutation (P451L) within the cytoplasmic domain of the receptor. Transfection studies in HEK cells showed that the P451L mutation severely affects P2X7 functions like pore formation and calcium flux in response to ATP in comparison to the wild type [63]. It seemed likely, therefore, that the P451L mutation in P2X7 accounted for the resistance to NAD-induced apoptosis in T lymphocytes from C57BL/6 mice. The P451L mutation in the mouse is reminiscent of a naturally occurring E496A mutation in the cytoplasmic domain of the human P2X7, which similarly affects the function of the receptor [64].

Collectively, these studies identify ADP-ribosylation of $\mathrm{P} 2 \mathrm{X} 7$ as an alternative way of activating P2X7 in $\mathrm{T}$ lymphocytes [39]. Although P2X7 has been implicated in important cellular functions, it is not fully clear how it is activated in vivo. Near millimolar concentrations of exogenous ATP are required to elicit $\mathrm{P} 2 \mathrm{X} 7$ activation in vitro $[65,66]$. In this context, ART2-catalysed ADPribosylation, requiring only micromolar concentrations of NAD, represents an appealing alternative pathway for the activation of P2X7. NAD-dependent activation of P2X7 
constitutes a novel mechanism for inducing $\mathrm{T}$ lymphocyte apoptosis. It is of note that this mechanism only affects resting $\mathrm{T}$ lymphocytes [39], consistent with the observation that ART2 is shed from the cell surface by the action of a metalloproteinase following T-cell activation [67]. Other known apoptosis-triggering pathways in $\mathrm{T}$ lymphocytes affect either immature $\mathrm{T}$ cells during their development in the thymus, or activated mature $\mathrm{T}$ lymphocytes, a process known as AICD (activation-induced cell death). NAD-induced apoptosis or NICD (NAD-induced cell death) is the first apoptosis pathway described affecting naive $\mathrm{T}$ lymphocytes.

P2X7 is also expressed on macrophages and dendritic cells. Recent studies have underlined the central role of P2X7 in inflammation by controlling IL-1 $\beta$ maturation and release [42]. Furthermore, P2X7 may play a role in the elimination of intracellular pathogens such as mycobacteria or Chlamydia by promoting phagosome-lysosome fusion [43-45]. To date no clear evidence of ART expression on these cell types has been found. It is also not known yet whether P2X7 can be activated by soluble ARTs or by an ART present on the surface of an apposing cell. It thus presently remains unclear whether NAD-dependent activation of $\mathrm{P} 2 \mathrm{X} 7$ can also occur on these cells.

Activation of P2X7 by ATP or via NAD-dependent ADP-ribosylation shows important differences. First of all, activation via NAD begins at substrate concentrations around $1 \mu \mathrm{M}$ and increases in a dose-dependent manner. By contrast, ATP-mediated activation does not occur below a certain threshold concentration, which is dependent on the cell type and usually lies above $100 \mu \mathrm{M}$. Importantly, brief treatment with NAD leads to long lasting activation of P2X7, while treatment with ATP in the same conditions produces reversible effects $[39,42]$. These characteristics are compatible with the notion of a covalently bound (immobilised ADP-ribose in the case of NAD-mediated activation) versus a soluble (ATP) ligand.

\section{Endogenous sources of NAD and ATP}

Under normal conditions the concentrations of ATP and NAD in body fluids are below the concentrations required to induce P2X7-dependent apoptosis. The concentration of NAD for instance is reported to be maintained between 0.1 to $0.5 \mu \mathrm{M}$ in the serum of non-treated animals $[68,69]$. It is therefore plausible that extracellular nucleotides are released from intracellular sources, where they are present in higher concentrations. As mentioned above, three situations may lead to the release of nucleotides from intracellular compartments: (1) liberation of intracellular contents by dying cells, (2) exocytosis of nucleotide-containing granules and (3) diffusion of these molecules towards the extracellular space across membrane channels.
Experiments in vitro demonstrated that cell lysates contain amounts of NAD sufficient to induce ART2dependent apoptosis of T lymphocytes [39]. These data suggest indirectly that NAD may be released in vivo by dead cells resulting from accidental tissue damage or consequent to the activity of the immune system itself. Notably, T cells from ART2-/- mice were not affected in these experiments, indicating that - at least at the concentrations employed - the lysates did not contain sufficient quantities of ATP to trigger the P2X7 receptor. This is consistent with the observation that activation of P2X7 occurs at low doses of NAD, but requires a threshold concentration of ATP $[39,57]$.

The hypothesis that purines are actively secreted from living cells and function as neurotransmitters was first formulated by G. Burnstock in 1972 [70]. Since then, many groups have confirmed that ATP, together with other neurotransmitters, is actively released from pre-synaptic vesicles of so-called "purinergic" nerves [5]. Recently, a similar mechanism has also been described for the release of NAD. According to this report, NAD can be released in combination with ATP and other neurotransmitters from stimulated postganglionic nerve terminals connected to blood vessels and urinary bladder [7]. Outside the nervous system, other mechanisms can account for the release of purine nucleotides. For example, both NAD and ATP have been reported to be transported through Connexin43 gapjunction hemi-channels $[6,71]$. Evidence is accumulating that these channels preferentially open under conditions of metabolic or mechanical stress [72, 73]. In vitro, release of purine nucleotides can be induced by moderate osmotic shocks, application of shear forces and following mechanical stress. In all these cases, release of purines appears to be an active process that follows the increase of intracellular calcium concentration [74, 75]. Finally, extracellular ATP itself may be a signal for the release of further ATP through activation of the P2X7 purinoreceptor [76].

Evidence is accumulating linking the release of purine nucleotides into the extracellular compartment with inflammation or cellular stress. If purine nucleotides are preferentially released into the extracellular compartment under conditions of cellular stress, it is conceivable that the massive recruitment of polymorphonuclear neutrophils and macrophages into sites of inflammation, which results in oxidative stress, tissue damage and massive neutrophil death, may lead to the release of NAD by both lytic and non-lytic mechanisms. In this context it is interesting to note that the only direct demonstration to date of ADPribosylation occurring in vivo using endogenous NAD as a substrate stems from an inflammatory environment. In humans, the defensin HNP-1 has been reported to be 
ADP-ribosylated in the bronchoalveolar fluid of smokers [77]. This suggests that NAD might be released in situations leading to chronic airway inflammation.

\section{The regulation of purinergic signalling by nucleotide-catabolising enzymes}

The deleterious effect of the ADP-ribosylation of P2X7 receptors on naive $\mathrm{T}$ lymphocytes raises the question of whether this phenomenon also occurs under physiological conditions. In in vitro experiments, more than $70 \%$ of naive T lymphocytes are susceptible to NICD [39, 57]. Obviously, this does not reflect the situation in a normal mouse. In living organisms, a flurry of nucleotide-catabolising enzymes tightly regulate the concentration of extracellular NAD and ATP. The steady-state concentration of NAD in biological fluids thus results from the equilibrium between its release from intracellular stores and its degradation by NAD-catabolising enzymes.

In principle, extracellular NAD may be hydrolysed by the CD38 and CD157 NAD-glycohydrolases or by phosphodiesterases (E-NPPs) present on the membrane of several cells as well as in soluble form in biological fluids. CD38 is the major NAD-glycohydrolase/ADP-ribosyl cyclase present in the extracellular compartment [27]. Experiments in vitro point to a major role of $\mathrm{CD} 38$ in controlling the level of ADP-ribosylation on the surface of $\mathrm{T}$ cells [26]. In these experiments, the presence of CD38, which is highly expressed by B cells, and at lower level on activated $\mathrm{T}$ cells, greatly impaired the level of ADPriboyslation detected on the surface of $\mathrm{T}$ cells upon treatment with NAD. Magnetic depletion of B cells from cell preparations and/or the use of ara-F-NAD to selectively block CD38 activity, greatly enhanced detectable ADPribosylation. In line with these findings, cells prepared from CD38-/- deficient mice showed increased apparent ADPribosyl transferase activity in vitro. These observations underline the important role of the CD38 NADase in regulating ADP-ribosylation reactions by limiting the concentration of available extracellular NAD. However, the situation may be more complex in vivo, where other NADmetabolising enzymes such as phosphodiesterases, which are expressed for instance on vascular endothelium, may also play a role to control the level of NAD in body fluids.

In a similar fashion, it has been shown that the CD39 ecto-nucleotidase is the major determinant for the regulation of extracellular ATP levels in blood. CD39 is also highly expressed on Langerhans cells of the skin, where it prevents hyperreactivity of these cells to ATP released from keratinocytes, for instance during injury by topically applied irritant chemicals [78]. Interestingly, CD39-deficient mice also show reduced T-cell dependent immune responses to antigens applied to the skin, pointing to as yet undefined modulating effects of ATP during the generation of these responses [78].

\section{The biological significance of NAD-mediated signalling}

Although several target proteins for ART2 have been identified, the activation of the $\mathrm{P} 2 \mathrm{X} 7$ purinoreceptor is presently the best-studied example of the functional consequences of mono-ADP-ribosylation. NAD-dependent, ART-mediated ADP-ribosylation represents an alternate mechanism of P2X7 activation that differs from "classical" activation by ATP in important aspects.

What may be the biological significance of NADmediated activation of P2X7? Importantly, it focuses P2X7 activation on a special population of cells, i.e., those that express both P2X7 and ART2. This is essentially the population of naive or resting T lymphocytes, since ART2 expression is limited to T cells, and activation of these cells leads to the proteolytic cleavage of ART2 by metalloproteases and its shedding from the cell surface as an active enzyme [67]. It is not yet clear whether the ART2 enzyme liberated from activated T cells is capable of ADPribosylating other target proteins that may be soluble or on the surface of other cells. Cells that have shed their ART2, however, are resistant to NAD-mediated activation of P2X7 and thus insensitive to NICD.

For these cells NAD-dependent P2X7 activation provides a mechanism for signalling through $\mathrm{P} 2 \mathrm{X} 7$ that requires only low concentrations of extracellular nucleotide, which may be more easily attainable in vivo than the high concentrations required for ATP-mediated signalling.

The precise role of NICD in vivo remains elusive. However, based on the available data, one can speculate that NICD is a mechanism to focus immune reactivity onto appropriate targets, i.e., pathogens causing tissue damage, while at the same time protecting against auto-immune reactions. According to this hypothesis, NAD is preferentially released at sites of tissue damage and inflammation, and would act locally to eliminate regulatory $\mathrm{T}$ cells, while sparing antigen-specific effector cells. This would result in an augmentation of the immune response at a site of infection. In addition, NICD would affect naïve $\mathrm{T}$ cells present in the local environment, thereby limiting the unwanted activation of bystander cells. Finally, it is conceivable that NAD might gain access to the draining lymph nodes in concentrations sufficient to elicit a cytotoxic effect. Here it would also act to augment the immune response. Besides killing regulatory $\mathrm{T}$ cells present in the lymph node, NAD could eliminate a fraction of naive $\mathrm{T}$ lymphocytes, thus creating space for the expansion of activated and memory lymphocytes. 


\section{Conclusions}

Extracellular nucleotides such as ATP and NAD are ideally suited as extracellular signal transmitters, since they can be rapidly mobilised from intracellular stores and their action is rapidly terminated by degradation through nucleotide catabolising enzymes. ATP and NAD are preferentially released from intracellular stores in conditions of cell stress or inflammation, and thus may function as classical "danger signals" to alert the immune response. A high degree of plasticity is attained by their capacity to signal through different receptors at different concentrations, as well as by their degradation to metabolites that by themselves are capable of signal transmission through other receptors. Consequently, the net effect of signalling in a given microenvironment will be critically dependent on the locally available nucleotide concentration and the particular constellation of nucleotide receptors and nucleotide-utilising enzymes present. Understanding the intricacies of the network of purine sensors on the surface of immune cells remains a major challenge that will ultimately lead to the development of new possibilities for pharmacological modulation of immune responses.

Note added in proof A recent study shows that P2Y11 can be activated by micromolar concentrations of ecto-NAD. Moreschi I et al (2006) Extracellular NAD + is an agonist of the human P2Y11 purinergic receptor in human granulocytes. J Biol Chem 281:31419-29.

Acknowledgements This work was supported by DFG grant No310/6-1 to FKN and FH, and grants from the Ligue Nationale Contre le Cancer, the Association pour la Recherche sur le Cancer, and the Ministère de la Recherche to MS. SA was a recipient of stipends from the Fondation pour la Recherche Medicale and the INSERM/DFG.

\section{References}

1. Berger F, Lau C, Dahlmann M et al (2005) Subcellular compartmentation and differential catalytic properties of the three human nicotinamide mononucleotide adenylyltransferase isoforms. J Biol Chem 280:36334-36341

2. D'Amours D, Desnoyers S, D'Silva I et al (1999) Poly(ADPribosyl)ation reactions in the regulation of nuclear functions. Biochem J 342:249-268

3. Herceg Z, Wang ZQ (2001) Functions of poly(ADP-ribose) polymerase (PARP) in DNA repair, genomic integrity and cell death. Mutat Res 477:97-110

4. Di Lisa F, Ziegler M (2001) Pathophysiological relevance of mitochondria in NAD $(+)$ metabolism. FEBS Lett 492:4-8

5. Schwiebert EM, Zsembery A (2003) Extracellular ATP as a signaling molecule for epithelial cells. Biochim Biophys Acta 1615:7-32

6. Bruzzone S, Guida L, Zocchi E et al (2001) Connexin 43 hemi channels mediate $\mathrm{Ca} 2+$-regulated transmembrane $\mathrm{NAD}+$ fluxes in intact cells. Faseb J 15:10-12

7. Smyth LM, Bobalova J, Mendoza MG et al (2004) Release of beta-nicotinamide adenine dinucleotide upon stimulation of postganglionic nerve terminals in blood vessels and urinary bladder. J Biol Chem 279:48893-48903

8. la Sala A, Ferrari D, Di Virgilio F et al (2003) Alerting and tuning the immune response by extracellular nucleotides. J Leukoc Biol 73:339-343

9. Hanley PJ, Musset B, Renigunta V et al (2004) Extracellular ATP induces oscillations of intracellular $\mathrm{Ca} 2+$ and membrane potential and promotes transcription of IL-6 in macrophages. Proc Natl Acad Sci U S A 101:9479-9484

10. Di Virgilio F (2005) Purinergic mechanism in the immune system: a signal of danger for dendritic cells. Purinergic Signalling 1:205-209

11. Ralevic V, Burnstock G (1998) Receptors for purines and pyrimidines. Pharmacol Rev 50:413-492

12. Dubyak GR (2003) Knock-out mice reveal tissue-specific roles of P2Y receptor subtypes in different epithelia. Mol Pharmacol 63:773-776

13. Fredholm BB, Abbracchio MP, Burnstock G et al (1997) Towards a revised nomenclature for $\mathrm{P} 1$ and $\mathrm{P} 2$ receptors. Trends Pharmacol Sci 18:79-82

14. Khakh BS, Burnstock G, Kennedy C et al (2001) International union of pharmacology. XXIV. Current status of the nomenclature and properties of $\mathrm{P} 2 \mathrm{X}$ receptors and their subunits. Pharmacol Rev 53:107-118

15. Burnstock G, Williams M (2000) P2 purinergic receptors: modulation of cell function and therapeutic potential. J Pharmacol Exp Ther 295, 862-869

16. Bodin P, Burnstock G (2001) Purinergic signalling: ATP release. Neurochem Res 26:959-969

17. Ferrari D, Chiozzi P, Falzoni S et al (1997) Purinergic modulation of interleukin-1 beta release from microglial cells stimulated with bacterial endotoxin. J Exp Med 185:579-582

18. Mehta VB, Hart J, Wewers MD (2001) ATP-stimulated release of interleukin (IL)-1beta and IL-18 requires priming by lipopolysaccharide and is independent of caspase-1 cleavage. J Biol Chem 276:3820-3826

19. Suzuki T, Hide I, Ido K et al (2004) Production and release of neuroprotective tumor necrosis factor by $\mathrm{P} 2 \mathrm{X} 7$ receptor-activated microglia. J Neurosci 24:1-7

20. la Sala A, Sebastiani S, Ferrari D et al (2002) Dendritic cells exposed to extracellular adenosine triphosphate acquire the migratory properties of mature cells and show a reduced capacity to attract type $1 \mathrm{~T}$ lymphocytes. Blood 99:1715-1722

21. la Sala A, Ferrari D, Corinti S et al (2001) Extracellular ATP induces a distorted maturation of dendritic cells and inhibits their capacity to initiate Th1 responses. J Immunol 166:1611-1617

22. Berthelier V, Tixier JM, Muller-Steffner H et al (1998) Human $\mathrm{CD} 38$ is an authentic $\mathrm{NAD}(\mathrm{P})+$ glycohydrolase. Biochem $\mathrm{J}$ 330:1383-1390

23. Aarhus R, Graeff RM, Dickey DM et al (1995) ADP-ribosyl cyclase and CD38 catalyze the synthesis of a calcium-mobilizing metabolite from NADP. J Biol Chem 270:30327-30333

24. Guse AH (2000) Cyclic ADP-ribose. J Mol Med 78:26-35

25. De Flora A, Franco L, Guida L et al (1998) Ectocellular CD38catalyzed synthesis and intracellular $\mathrm{Ca}(2+)$ - mobilizing activity of cyclic ADP-ribose. Cell Biochem Biophys 28:45-62

26. Krebs C, Adriouch S, Braasch F et al (2005) CD38 controls ADPribosyltransferase-2-catalyzed ADP-ribosylation of T cell surface proteins. J Immunol 174:3298-3305

27. Cockayne DA, Muchamuel T, Grimaldi JC et al (1998) Mice deficient for the ecto-nicotinamide adenine dinucleotide glycohydrolase CD38 exhibit altered humoral immune responses. Blood 92:1324-1333

28. Partida-Sanchez S, Goodrich S, Kusser K et al (2004) Regulation of dendritic cell trafficking by the ADP-ribosyl cyclase CD38: impact on the development of humoral immunity. Immunity 20:279-291 
29. Koch-Nolte F, Petersen D, Balasubramanian S et al (1996) Mouse T cell membrane proteins Rt6-1 and Rt6-2 are arginine/protein mono(ADPribosyl)transferases and share secondary structure motifs with ADP-ribosylating bacterial toxins. J Biol Chem 271:7686-7693

30. Glowacki G, Braren R, Firner K et al (2002) The family of toxinrelated ecto-ADP-ribosyltransferases in humans and the mouse. Protein Sci 11:1657-1670

31. Koch-Nolte F, Glowacki G, Bannas P et al (2005) Use of genetic immunization to raise antibodies recognizing toxin-related cell surface ADP-ribosyltransferases in native conformation. Cell Immunol 236:66-71

32. Yadollahi-Farsani M, Kefalas P, Saxty BA et al (1999) Polymorphic forms of human ADP-ribosyltransferase-1 differences in their catalytic activities revealed by labeling of membraneassociated substrates. Eur J Biochem 262:342-348

33. Zolkiewska A, Moss J (1993) Integrin alpha 7 as substrate for a glycosylphosphatidylinositol-anchored ADP-ribosyltransferase on the surface of skeletal muscle cells. J Biol Chem 268:25273-25276

34. Zhao Z, Gruszczynska-Biegala J, Zolkiewska A (2005) ADPribosylation of integrin alpha7 modulates the binding of integrin alpha7beta1 to laminin. Biochem J 385:309-317

35. Parusel I, Kahl S, Braasch F et al (2005) A panel of monoclonal antibodies recognizing GPI-anchored ADP-ribosyltransferase ART4, the carrier of the Dombrock blood group antigens. Cell Immunol 236:59-65

36. Koch-Nolte F, Reche P, Haag F et al (2001) ADP-ribosyltransferases: plastic tools for inactivating protein and small molecular weight targets. J Biotechnol 92:81-87

37. Bannas P, Adriouch S, Kahl S et al (2005) Activity and specificity of toxin-related mouse $\mathrm{T}$ cell ecto-ADP-ribosyltransferase ART2.2 depends on its association with lipid rafts. Blood 105:3663-3670

38. Di Virgilio F, Chiozzi P, Ferrari D et al (2001) Nucleotide receptors: an emerging family of regulatory molecules in blood cells. Blood 97:587-600

39. Seman M, Adriouch S, Scheuplein F et al (2003) NAD-induced T cell death: ADP-ribosylation of cell surface proteins by ART2 activates the cytolytic P2X7 purinoceptor. Immunity 19:571-582

40. Somers GR, Hammet FM, Trute L et al (1998) Expression of the $\mathrm{P} 2 \mathrm{Y} 6$ purinergic receptor in human T cells infiltrating inflammatory bowel disease. Lab Invest 78:1375-1383

41. Scrivens M, Dickenson JM (2005) Functional expression of the $\mathrm{P} 2 \mathrm{Y} 14$ receptor in murine T-lymphocytes. $\mathrm{Br} \mathrm{J}$ Pharmacol $146: 435-444$

42. MacKenzie A, Wilson HL, Kiss-Toth E et al (2001) Rapid secretion of interleukin-1 beta by microvesicle shedding. Immunity $15: 825-835$

43. Lammas DA, Stober C, Harvey CJ et al (1997) ATP-induced killing of mycobacteria by human macrophages is mediated by purinergic $\mathrm{P} 2 \mathrm{Z}(\mathrm{P} 2 \mathrm{X} 7)$ receptors. Immunity 7:433-444

44. Fairbairn IP, Stober CB, Kumararatne DS et al (2001) ATPmediated killing of intracellular mycobacteria by macrophages is a P2X(7)-dependent process inducing bacterial death by phagosome-lysosome fusion. J Immunol 167:3300-3307

45. Coutinho-Silva R, Stahl L, Raymond MN et al (2003) Inhibition of chlamydial infectious activity due to P2X7R-dependent phospholipase D activation. Immunity 19:403-412

46. Adinolfi E, Melchiorri L, Falzoni S et al (2002) P2X7 receptor expression in evolutive and indolent forms of chronic B lymphocytic leukemia. Blood 99:706-708

47. Wiley JS, Dao-Ung LP, Gu BJ et al (2002) A loss-of-function polymorphic mutation in the cytolytic $\mathrm{P} 2 \mathrm{X} 7$ receptor gene and chronic lymphocytic leukaemia: a molecular study. Lancet 359:1114-1119
48. Wilkin F, Duhant X, Bruyns C et al (2001) The P2Y11 receptor mediates the ATP-induced maturation of human monocytederived dendritic cells. J Immunol 166:7172-7177

49. Wilkin F, Stordeur P, Goldman M et al (2002) Extracellular adenine nucleotides modulate cytokine production by human monocyte-derived dendritic cells: dual effect on IL-12 and stimulation of IL-10. Eur J Immunol 32:2409-2417

50. Schnurr M, Toy T, Stoitzner P et al (2003) ATP gradients inhibit the migratory capacity of specific human dendritic cell types: implications for P2Y11 receptor signaling. Blood 102: 613-620

51. Panther E, Idzko M, Herouy Y et al (2001) Expression and function of adenosine receptors in human dendritic cells. Faseb J 15: 1963-1970

52. Panther E, Corinti S, Idzko $M$ et al (2003) Adenosine affects expression of membrane molecules, cytokine and chemokine release, and the T-cell stimulatory capacity of human dendritic cells. Blood 101:3985-3990

53. Huang S, Apasov S, Koshiba M et al (1997) Role of A2a extracellular adenosine receptor-mediated signaling in adenosinemediated inhibition of T-cell activation and expansion. Blood 90:1600-1610

54. Koshiba M, Kojima H, Huang S et al (1997) Memory of extracellular adenosine A2A purinergic receptor-mediated signaling in murine T cells. J Biol Chem 272:25881-25889

55. Sitkovsky M, Lukashev D (2005) Regulation of immune cells by local-tissue oxygen tension: HIF1 alpha and adenosine receptors. Nat Rev Immunol 5:712-721

56. Yegutkin GG, Henttinen T, Samburski SS et al (2002) The evidence for two opposite, ATP-generating and ATP-consuming, extracellular pathways on endothelial and lymphoid cells. Biochem J 367:121-128

57. Adriouch S, Ohlrogge W, Haag F et al (2001) Rapid induction of naive $\mathrm{T}$ cell apoptosis by ecto-nicotinamide adenine dinucleotide: requirement for mono(ADP-ribosyl)transferase 2 and a downstream effector. J Immunol 167:196-203

58. Aswad F, Kawamura H, Dennert G (2005) High sensitivity of $\mathrm{CD} 4+\mathrm{CD} 25+$ regulatory $\mathrm{T}$ cells to extracellular metabolites nicotinamide adenine dinucleotide and ATP: a role for P2X7 receptors. J Immunol 175:3075-3083

59. Ohlrogge W, Haag F, Lohler J et al (2002) Generation and characterization of ecto-ADP-ribosyltransferase ART2.1/ART2.2deficient mice. Mol Cell Biol 22:7535-7542

60. Krebs C, Koestner W, Nissen M et al (2003) Flow cytometric and immunoblot assays for cell surface ADP-ribosylation using a monoclonal antibody specific for ethenoadenosine. Anal Biochem 314:108-115

61. Di Virgilio F, Chiozzi P, Falzoni S et al (1998) Cytolytic P2X purinoceptors. Cell Death Differ 5:191-199

62. Di Virgilio F (1998) ATP as a death factor. Biofactors 8: 301303

63. Adriouch S, Dox C, Welge V et al. (2002) Cutting edge: a natural P451L mutation in the cytoplasmic domain impairs the function of the mouse P2X7 receptor. J Immunol 169:4108-4112

64. Gu BJ, Zhang W, Worthington RA et al (2001) A Glu-496 to Ala polymorphism leads to loss of function of the human P2X7 receptor. J Biol Chem 276:11135-11142

65. Surprenant A, Rassendren F, Kawashima E et al (1996) The cytolytic P2Z receptor for extracellular ATP identified as a P2X receptor (P2X7). Science 272:735-738

66. North RA, Surprenant A (2000) Pharmacology of cloned P2X receptors. Annu Rev Pharmacol Toxicol 40:563-580

67. Kahl S, Nissen M, Girisch R et al (2000) Metalloproteasemediated shedding of enzymatically active mouse ecto-ADPribosyltransferase ART2.2 upon $\mathrm{T}$ cell activation. J Immunol $165: 4463-4469$ 
68. Davies CA, Perrett D, Zhang Z et al (1999) Simultaneous analysis of nitrite, nitrate and the nicotinamide nucleotides by capillary electrophoresis: application to biochemical studies and human extracellular fluids. Electrophoresis 20:2111-2117

69. O'Reilly T, Niven DF (2003) Levels of nicotinamide adenine dinucleotide in extracellular body fluids of pigs may be growthlimiting for Actinobacillus pleuropneumoniae and Haemophilus parasuis. Can J Vet Res 67:229-231

70. Burnstock G (1972) Purinergic nerves. Pharmacol Rev 24:509-581

71. Stout CE, Costantin JL, Naus CC et al (2002) Intercellular calcium signaling in astrocytes via ATP release through connexin hemichannels. J Biol Chem 277:10482-10488

72. Contreras JE, Sanchez HA, Eugenin EA et al (2002) Metabolic inhibition induces opening of unapposed connexin 43 gap junction hemichannels and reduces gap junctional communication in cortical astrocytes in culture. Proc Natl Acad Sci U S A 99:495-500

73. Cherian PP, Siller-Jackson AJ, Gu S et al (2005) Mechanical strain opens connexin 43 hemichannels in osteocytes: a novel mechanism for the release of prostaglandin. Mol Biol Cell 16:3100-3106

74. Boudreault F, Grygorczyk R (2004) Cell swelling-induced ATP release is tightly dependent on intracellular calcium elevations. J Physiol 561:499-513

75. Genetos DC, Geist DJ, Liu D et al (2005) Fluid Shear-Induced ATP Secretion Mediates Prostaglandin Release in MC3T3-E1 Osteoblasts. J Bone Miner Res 20:41-49

76. Pellegatti P, Falzoni S, Pinton P et al (2005) A novel recombinant plasma membrane-targeted luciferase reveals a new pathway for ATP secretion. Mol Biol Cell 16:3659-3665

77. Paone G, Wada A, Stevens LA et al (2002) ADP ribosylation of human neutrophil peptide-1 regulates its biological properties. Proc Natl Acad Sci U S A 99:8231-8235

78. Mizumoto N, Kumamoto T, Robson SC et al (2002) CD39 is the dominant Langerhans cell-associated ecto-NTPDase: modulatory roles in inflammation and immune responsiveness. Nat Med $8: 358-365$ 